\title{
Invited Commentary Special Section: Addressing the Needs of Official Statistics Users: The Case of Eurostat
}

\author{
Dr. Marleen De Smedt ${ }^{1}$
}

As the producer of official statistics for the European Union, Eurostat aims to produce relevant and accurate European statistics (official statistics at the European level) that are directly accessible to and understood and used by a wide range of users.

On a monthly basis, the Eurostat website records more than three million visits, over four million page views, some 700,000 pdf downloads and more than one million extractions of data. These metrics rank the site among the top five websites of the European Commission (Bautier et al. 2014). But who are these users? And what are they looking for?

Eurostat obtains information about the users of its data in various ways: via bilateral contacts with Commission policy services (via "annual hearings" and through written agreements - "Memorandum of Understanding"), via requests that users address to the Eurostat Statistical Support services (which include the Eurostat User Support network and separate central services for media and institutional requests) and via the annual Eurostat User Satisfaction Surveys.

The Eurostat User Support network consists of Eurostat and Support Centres in all 28 EU countries and in a number of countries in the wider European area. This network has been in operation since 2004. Users can put forward specific questions to this network, which in turn gives a first insight of the type of users and the main type of support wanted.

In the period April 2015 to September 2015, the Eurostat User Support network processed 3,757 requests and, in the same period, 1,271 requests were sent directly to Eurostat. Of these requests sent directly to Eurostat a further analysis was made on the type of user (see Table 1).

Through an annual Eurostat User Satisfaction Survey, which includes general questions about clarity and user-friendliness of the data, Eurostat is reaching out to its users - inside and outside the Commission services - in order to learn about the type of users and uses, users' assessment of the quality of, and trust in, European statistics and on its dissemination.

The report on the 2015 User Satisfaction Survey indicated that students, academic and private users account for $43.5 \%$ of users and that "research" and "general background information" were the most common purposes for all users combined. Results also showed a high user satisfaction rate with the renewed Eurostat website, a significant improvement

${ }^{1}$ Advisor to the Director-General, Eurostat, European Commission, Luxembourg. Email: marleen.desmedt@ ec.europa.eu 


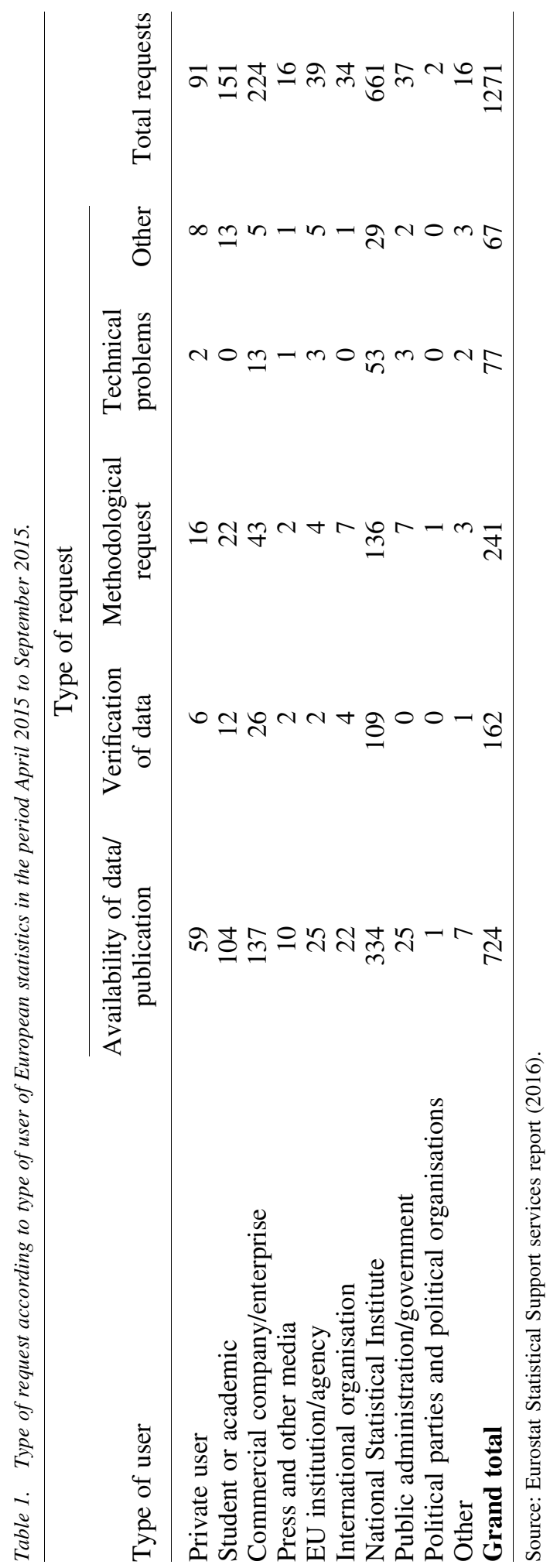


in ease of understanding European statistics, and a high level of trust among users of European statistics.

Previous User Satisfaction Surveys (before 2014) indicated that many users wanted easier access to data, "ready-to-use" statistical tables and more context information. This has resulted in continuous improvements of data presentation on the Eurostat website, which now offers - in addition to the detailed data base and extraction tools - a series of most popular tables, key figures and domains in focus (i.e., "Sustainable Development" and "GDP and beyond") as well as Statistics Explained.

Statistics Explained is a special website that uses Wiki techniques and presents a number of articles that make up an encyclopedia of European statistics for everyone, supplemented by a statistical glossary clarifying all terms used and by numerous links to further information. It is a portal for occasional as well as for regular users. From January to July in 2015, Statistics Explained had over three million page views with a high current number of visits and rising. For specific domains, such as "quality of life", "you in the EU", "young Europeans" users can go directly from Eurostat's main page to "Infographics", which presents statistics in a user-friendly way. In addition, mobile apps can be downloaded (for instance concerning the EU economy and country profiles).

In addition, Eurostat has invested in organising training sessions for Commission policy departments and provides practical training on how to access, interpret and use statistics to groups of visitors and journalists. The European Statistical Training Programme (ESTP), coordinated by Eurostat, helps officials and employees of the ESS improve statistical literacy, taking into account the different levels of statistical knowledge and working experiences. It includes courses in Official Statistics, IT applications, Research and Development and Statistical Management. As a result of another programme for Training and Education in Official Statistics within existing Master programmes at European universities, the European Statistical System Committee (ESSC) awarded - in May 2015

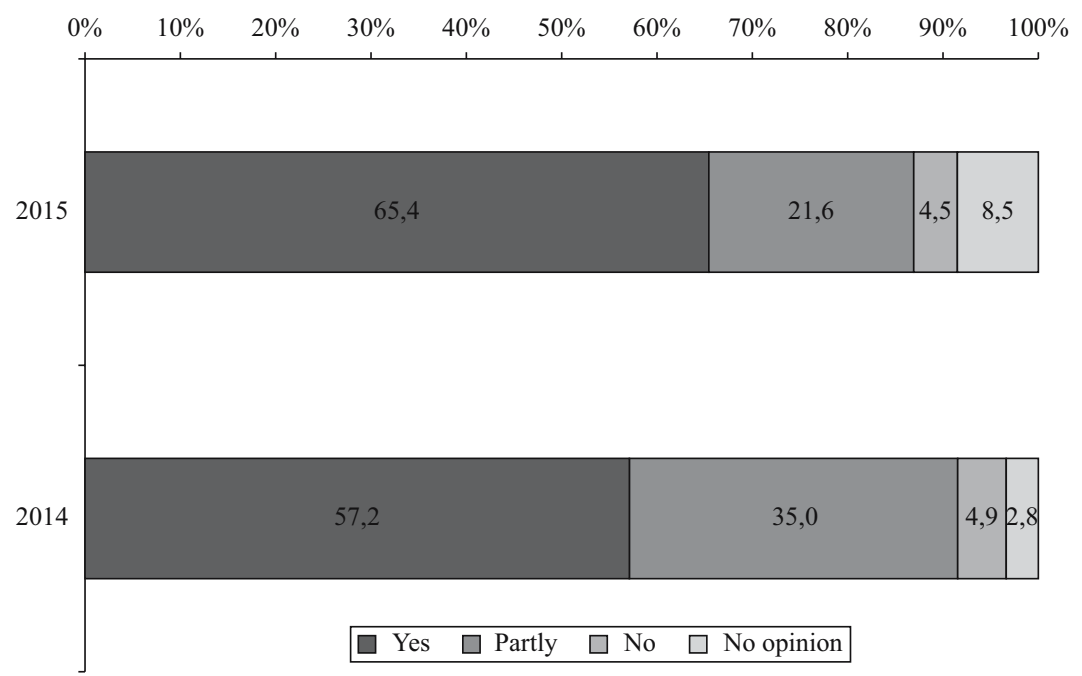

Fig. 1. Assessment of the presentation of the statistics on the website, in \%. (Are European statistics presented in an easy-to-understand way?) 
for the first time - twelve university master programmes with the European Master in Official Statistics (EMOS) label.

The 2015 User Satisfaction Survey showed an important rise in the percentage of users satisfied with the presentation of Eurostat data (Figure 1).

These are satisfactory results, but there is still room for continued efforts to improve clarity and accessibility of European statistics. In addition, the data environment is constantly evolving with new openings such as open data, big data, and use of geographic information.

To respond to these challenges, in 2014 Eurostat and its partners in the ESS agreed on a Vision 2020 priority area for action on "Identifying user needs and cooperation with stakeholders" (European Statistical System Committee 2014). In 2015, a statistical project, DIGICOM, was launched among the ESS partners to support their actions on user analysis and on creating a new dissemination and communication strategy using innovative technologies.

Users of European statistics can also reach through to the European official statistics providers within the framework of the biannual meetings of the European Statistical Advisory Committee (ESAC). This Committee has set up a "classification" of users, thereby allowing producers of European statistics to focus on different sets of users and different contexts (Vichi et al. 2015).

The above illustrates Eurostat's efforts aimed at satisfying the needs of its users. However, before any further steps in this direction are taken it might be wise to first thoroughly assess statistical literacy of the current groups of users to better target their needs - now and in the future. This assessment should also include user groups represented by official statisticians and by small and medium enterprises (SMEs) - as discussed by Deutsch, Forbes and Keegan, as well as Coleman in the special section of this issue.

\section{References}

Bautier, P., C. Laevaert, and B. Le Goff. 2014. Tracking Users for a Targeted Dissemination, Paper presented at the Conference of European Statistics Stakeholders in Rome, 2015.

European Statistical System Committee. 2014. Vision2020. Available at: http://ec.europa. eu/eurostat/web/ess/about-us/ess-vision-2020 (accessed September 2016).

Vichi, M., M. João Valente Rosa, and F. Ruane. 2015. The Users of Statistics and Their Role in the European Society, ESAC document 2015/1175; http://ec.europa.eu/eurostat/ web/european-statistical-advisory-committee-esac/activities/esac-documents (accessed September 2016). 